THE JEFFERSON METHOD OF APPORTIONMENT *

\author{
M. L. Balinski ** \\ H.P. Young ${ }^{\star \star}$
}

August 1976

Professional Papers are not official publications of the International Institute for Applied Systems Analysis, but are reproduced and distributed by the Institute as an aid to staff members in furthering their professional activities. Views or opinions expressed herein are those of the author and should not be interpreted as representing the view of either the Institute or the National Member Organizations supporting the Institute.

\title{
*
}

This work was supported by the National Science Foundation under Contract MCS 75-07414 AOl with the Graduate Center of the City University of New York.

$\star \star$

Graduate School and University Center, City University of New York and International Institute for Applied Systems Analysis, Laxenburg, Austria. 



\section{The Jefferson Method of Apportionment}

M.L. Balinski and H.P. Young

\section{Abstract}

The Jefferson method of apportionment is characterized by three properties: consistency, housemonotonicity, and satisfying lower quota. The method of smallest devisors is characterized similarly by substituting upper quota for lower quota.

\section{The Congressional Apportionment Problem}

Let $\underset{\sim}{p}=\left(p_{1}, \ldots, p_{s}\right)$ be the populations of $s$ states, $p_{i}>0$ for all $i$, and $h \geqq 0$ be the number of seats in the House of Representatives. The problem is to find an apportionment for $h$, $a=\left(a, \ldots, a_{s}\right)$ each $a_{i} \geqq 0$ and integer, $\sum_{1}^{s} a_{i}=h$, which is, as. required by the Constitution, "according to their [the states'] respective numbers," that is, which is in some sense proportional to the populations $\underset{\sim}{\mathrm{p}}$ of the respective states.

An apportionment solution is a function $\underset{\sim}{f}$ which to any population vector and all $\mathrm{h}$ associates a unique apportionment for $\mathrm{h}$, $a_{i}=f_{i}(p, h)$ with $\sum_{i} a_{i}=h$. If $\underset{\sim}{f}$ is a solution and $h$ a house size then ${\underset{f}{\sim}}^{h}$ is the function restricted to the domain $\left(\underset{\sim}{p}, h^{\prime}\right)$, where

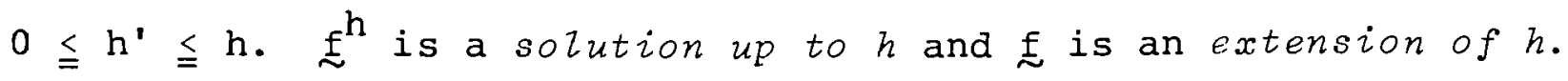

Different solutions to a problem must be admitted since "ties" may occur, such as when two states with identical populations must share an odd number of seats. Accordingly, an 
apportionment method $\underset{\sim}{\mathrm{M}}$ is defined to be a non-empty set of solutions. A specific solution up to some $h$ may have several different extensions.

2. The Jefferson Proposal of 1792

The first apportionment bill (based upon the census of 1790), heatedly discussed and amended, was passed by Congress on 23 March 1792. George Washington vetoed it on 5 April 1792 primarily because, "...there is no one proportion or divisor which, applied to the respective numbers of the states, will yield the number and allotment of Representatives proposed by the bill [8]."

The population figures and first bill's apportionment are given in Table 1. Washington sought out the opinions of Hamilton, Jefferson, Knox and Randolph, and followed the advice of Jefferson, with the concordance of Knox and Randolph, in casting this first Presidential veto. But Hamilton supported the bill, for, as he correctly maintained, it resulted from applying the following, seemingly reasonable method [5]. Define the exact quota of state $j$ to be $\left.q_{j} \underset{\sim}{p}, h\right)=p_{j} h / \Sigma_{i} p_{i}$. Then, Hamilton's method is: first, give to each state $i$ the integer part of its exact quota $\left\lfloor q_{i}\right\rfloor$, and order the states by their fractional remainders $d_{j}=q_{j}-\left\lfloor q_{j}\right\rfloor$ in a priority 1 ist $d_{j_{1}} \geqq \cdots \geqq d_{j_{s}}$. Second, give one additional seat to each of the first $h-\Sigma_{i}\left\lfloor q_{i}\right\rfloor=\Sigma_{j} d_{j}$ states on the list.

Jefferson [6], in disagreeing with Hamilton, said, "I answer, then, that taxes must be divided exactly, and representatives 
as nearly as the nearest ratio will admit; and the fractions must be neglected, because the Constitution calls absolutely that there be an apportionment or common ratio, and if any fractions result from the operation, it has left them unprovided for." Let $\lfloor\lfloor x\rfloor\rfloor$ be the greatest integer less than $x$ if $x$ is noninteger, and otherwise be equal to $x$ or $x-1$.

For a given $h, \Sigma_{i} p_{i} / h=\lambda *$ represents the average size of a district, i.e. a "common ratio." Jefferson's proposal is: choose a ratio $\lambda\left(\leqq \lambda^{*}\right)$ such that $\Sigma_{i}\left[\left[\mathrm{p}_{j} / \lambda\right\rfloor\right]=h$ has a solution; then apportionments for $h$ are found by taking $a_{i}=\left\lfloor\left\lfloor p_{i} / \lambda\right\rfloor\right\rfloor$ satisfying $\Sigma_{i} a_{i}=h$. (The requirement that one take the "nearest" $\lambda$ having this property is superfluous.)

An attempt to override Washington's veto failed and a new bill was approved on 14 April 1792 in which a "common ratio" of $\lambda=33,000$ was chosen and Jefferson's proposal used, thus giving $h=105$. This solution is found in Table 1. In addition to arguing against Hamilton, Jefferson further objected "[the bill] seems to have avoided establishing that [Hamilton's method] into a rule, lest it might not suit on another occasion." Thus he clearly saw the need for a method. It happensithat Jeffersonds approach gave to Virginia 19 seats whereas Hamilton's would have given it on ly 18

Jefferson's proposal was actually used as the apportionment rule applied to the first six censuses. However, the users seemed unaware of the fact that they could choose the size of the House h first, then obtain the proper $\lambda$. Rather, they typically debated hordes of different $\lambda$ 's and took the h's these produced. 
Jefferson's approach may be viewed as a particular "rounding" procedure. Namely, choose a common divisor $\lambda$, and for each state compute $\mathrm{p}_{\mathrm{i}} / \lambda$ and round down to the nearest integer. Instead, one might consider finding apportionments by rounding $u p$. Specifically, let $[[x]]$ be the smallest integer greater than $x$ if $x$ is not integer, and otherwise equal to $x$ or $x+1$. Choose a $\lambda\left(\geqq \Sigma_{i} p_{i} / h\right)$ such that $\Sigma_{i}\left\lceil\left\lceil p_{i} / \lambda\right\rceil\right\rceil=h$ can be obtained; then apportionments for $h$ found by taking $a_{i}=\left[\left[p_{i} / \lambda\right]\right]$ satisfying $\Sigma_{i} a_{i}=h$ lead to the "method of smallest divisors."

of course, another natural rounding procedure is to round to the nearest integer. Specifically, let [ $[\mathrm{x}]]$ be $\mathrm{x}$ rounded to the nearest integer if $x$ does not have a remainder of exactly $1 / 2$ and otherwise be equal to $x-1 / 2$ or $x+1 / 2$. Choose a $\lambda$ such that $\Sigma_{i}\left[\left[\mathrm{p}_{i} / \lambda\right]\right]=h$ can be obtained. Then apportionments for $h$ found by taking $a_{i}=\left[\overline{\left.\left[p_{i} / \lambda\right]\right]}=h\right.$ satisfying $\Sigma_{i} a_{i}=h$ lead to what we have called elsewhere "Webster's method" $[3,9]$. For the populations of 1790 and $h=105$ the Webster apportionment happen to be the same as Hamilton's.

\section{The Characterizations}

A desirable property of any apportionment method is that the number of seats accorded to any state not decrease if the house size increases. We say that a method $M$ is house monotone if for any $\underset{\sim}{\mathrm{M}}$-solution $\mathrm{f}$ and all $\underset{\sim}{\mathrm{p}}, \mathrm{h}$,

$$
E_{i}(\underline{\sim}, h+1) \geq E_{i}(\underset{\sim}{p}, h) \quad, \quad i=1,2, \ldots, s .
$$


Except for the case of ties, Jefferson's "method" is easily seen to be house monotone, since as $\mathrm{h}$ increases $\lambda$ decreases, whence the numbers $\left\lfloor\left\lfloor p_{i} / \lambda\right\rfloor\right\rfloor$ do not decrease. If ties occur, they can clearly always be resolved so that no state loses a seat in a larger house. Formally, we therefore define the Jefferson method $\mathrm{J}$, to be the set of all house monotone solutions that yield Jefferson apportionments. An easily computable characterization of all such solutions is given below in Lemma $1(b)$. By contrast, the Hamilton method does not yield a house monotone method, as many examples attest [3]. This fact was uncovered in the 1880's when the notorious "Alabama paradox" occurred.

A second basic property of methods revolves around the question: which state would most "deserve" to get an extra seat if there were one more seat to be distributed? This notion applies particularly to house monotone methods, since in going from any apportionment for $h$ to an apportionment for $h+1 a$ house monotone method gives the additional seat to exactly one state and all other allocations remain the same.

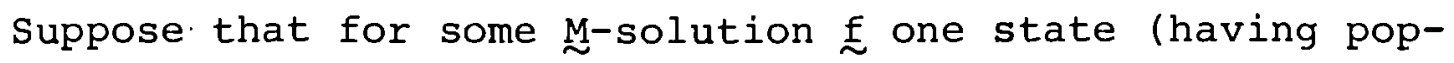
ulation $\bar{p}$ ) :has $\bar{a}$ seats at $\mathrm{h}$ while another state (having population $\mathrm{p}^{*}$ ) has a* seats, and $\underset{\sim}{\sim}$ allocates the $(\mathrm{h}+1)^{\text {st }}$ seat to the $\bar{p}$-state: then the bar-state is said to have weak priority over the star-state at $\left(\overline{\mathrm{p}}, \overline{\mathrm{a}} ; \mathrm{p}^{*}, \mathrm{a}^{*}\right)$, written $(\overline{\mathrm{p}}, \overline{\mathrm{a}}) \geq\left(\mathrm{p}^{*}, \mathrm{a}^{*}\right)$. If in another problem we also have that $\left(p^{*}, a^{*}\right) \geq(\bar{p}, \bar{a})$, then we say the bar-state and star-state are tied, written $(\bar{p}, \bar{a}) \sim\left(p^{*}, a^{*}\right)$. We say that the method $\underset{\sim}{\sim}$ is consistent if it treats tied states equally as regards receiving one more seat; 
that is, there must be an alternate $\underset{\sim}{\mathrm{M}}$-solution $\underset{\sim}{\mathfrak{f}}$ which is an extension of $f^{h}$ giving the $(h+1)^{\text {st }}$ seat to the star-state instead of the bar-state.

The Jefferson method is consistent (see Lemma 1(b)), as are the five Huntington or "modern" methods; in fact, it may be shown that the (generalized) "Huntington methods" are precisely the class of consistent, house-monotone methods [4]. However, the Hamilton method is not consistent as can be shown by example (see [2]).

While the Hamilton method fails to satisfy two basic properties of apportionment methods, it does have the distinct virtue that its apportionment solutions are "close" to the exact quotas-indeed the Hamilton method may be said to have been motivated by this desire. If $q_{i}$ is the exact quota of state $i$, let $\left\langle q_{i}\right\rfloor$ be the largest integer less than or equal to $q_{i}$ and $\left\lceil q_{i}\right\rceil$ the smallest integer bigger than or equal to $q_{i}$. Then $\mathbb{M}$ is said to satisfy Lower quota if for any $\underset{\sim}{\mathbb{M} \text {-apportionment }} \mathrm{a}, \mathrm{a}_{\mathrm{i}} \geqq\left\lfloor\mathrm{q}_{\mathrm{i}}\right\rfloor$, and $\underset{\mathcal{M}}{\mathrm{M}}$ satisfies upper quota if $a_{i} \leqq\left\lceil q_{i} l\right.$.

A method $\underset{\sim}{M}$ is said to be the unique method satisfying certain properties if any other method $\underset{\sim}{\mathcal{M}}$ having these properties is a set of $\underset{\sim}{M}-$ solutions, i.e. $\mathbb{M}^{\prime} \subseteq \mathbb{M}$.

Theorem 1. The Jefferson method is the unique method that is house monotone, consistent, and satisfies lower quota.

First we need the following Lemma.

Lemma 1. (a) Given $\underset{\sim}{\mathrm{p}}$ and $\mathrm{h}, \underset{\sim}{\mathrm{a}}=\left(\mathrm{a}_{1}, \ldots, \mathrm{a}_{\mathrm{s}}\right)$ is a Jefferson apportionment for $h$ if and only if

$$
\max _{i} p_{i} /\left(a_{i}+1\right) \leqq \min _{i} p_{i} / a_{i}
$$


(b) The set of Jefferson solutions is the set of all solutions $\underset{\sim}{f}$ obtained recursively as follows:

(i) $\underset{\sim}{ \pm}(\underline{p}, 0)=0$;

(ii) if $a_{i}=f_{i}(p, h)$ is an apportionment for $h$ and $k$ is some one state for which $p_{k} /\left(a_{k}+1\right)=\max _{i} p_{i} /\left(a_{i}+1\right)$ then

$$
\mathrm{f}_{\mathrm{k}}(\underset{\sim}{\mathrm{p}}, \mathrm{h}+1)=\mathrm{a}_{\mathrm{k}}+1, \quad \mathrm{f}_{i}=\left(\mathrm{p}_{i}, \mathrm{~h}+1\right)=\mathrm{a}_{i} \text { for } i \neq k \text {. }
$$

Proof. By definition, $\left(a_{1}, \ldots, a_{s}\right)$ is a Jefferson apportionment for $h$ if and only if $\Sigma a_{i}=h$ and for some $\lambda$

(1) $\quad a_{i}=\left\lfloor\left\lfloor p_{i} / \lambda\right\rfloor\right\rfloor$.

Now (1) holds if and only if

$$
a_{i}+1 \geq p_{i} / \lambda \geqq a_{i}
$$

equivalently,

$$
p_{i} /\left(a_{i}+1\right) \leqq \lambda \leqq p_{i} / a_{i} \text { for all } i \text {, }
$$

(where if $\left.a_{i}=0, p_{i} / a_{i}=+\infty\right)$, equivalently,

(2) $\max p_{i} /\left(a_{i}+1\right) \leqq \min p_{i} / a_{i}$,

proving (a). 
Given $\underset{\sim}{p}, \underset{\sim}{f}(p, 0)=0$ satisfies $(2)$. Suppose we have shown that any solution up through h obtained via (b) satisfies (2). Then clearly giving one more seat to some state $k$ maximizing $\mathrm{p}_{i} /\left(\mathrm{a}_{\mathrm{i}}+1\right)$ results in an apportionment also satisfying (2). Thus all solutions obtained via (b) satisfy inequality (2) and are house monotone, hence they are all Jefferson solutions. Conversely, suppose $\underset{\sim}{f}$ is a Jefferson method solution that is not obtained via (b). Then there is a solution $\underset{\sim}{g}$ obtained via (b)

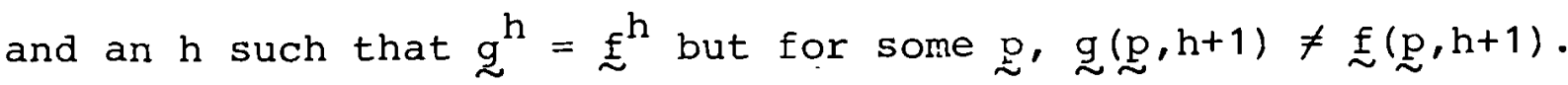
If $\left(a_{1}, \ldots, a_{s}\right)$ is the common allocation at $h$, $f$ must therefore accord the $(h+1)^{\text {st }}$ seat to some state $l$ such that $p_{\ell} /\left(a_{\ell}+1\right)<$ $\max _{i} p_{i} /\left(a_{i}+1\right)=p_{k} /\left(a_{k}+1\right)$, but then the new allocation does not satisfy (2), a contradiction. $\square$

For the proof of Theorem 1 we introduce the following technical notion. We say that a method $\underset{\sim}{\mathbb{M}}$ is balanced if whenever two states have the same population then their apportionments cannot differ by more than one.

\section{Proof of Theorem 1.}

The Jefferson method is house monotone--indeed it is given by the set of solutions defined by Lemma 1(b). Moreover, this characterization shows at once that it is consistent. To see that J satisfies lower quota, suppose not. Then for some apportionment $\underset{\sim}{\text { f for }} \mathrm{h}$ we have $\mathrm{a}_{i}<\left\lfloor\mathrm{q}_{i}\right\rfloor$, that is, $\mathrm{a}_{i} \leq \mathrm{q}_{i}-1$. Then for some state $j \neq i$ we must have $a_{j}>q_{j}$. Letting $\lambda=\Sigma_{k} p_{k} / h$ we have then that $q_{i}=p_{i} / \lambda, q_{j}=p_{j} / \lambda$ and 


$$
p_{j} / a_{j}<\lambda \leqq p_{i} /\left(a_{i}+1\right)
$$

contradicting Lemma $1(a)$.

Conversely, suppose that $\underset{\sim}{\mathbb{M}}$ is consistent house-monotone and satisfies lower quota, but is not a set of Jefferson solutions. Then by Lemma 1 (b) there exist populations $p, q$ having $\mathbb{M}-a 110-$ cations $\mathrm{a}$, b which are also Jefferson allocations, but

(3) $\quad(p, a) \gtrsim_{M}(q, b)$ whereas $p /(a+1)<q /(b+1)$.

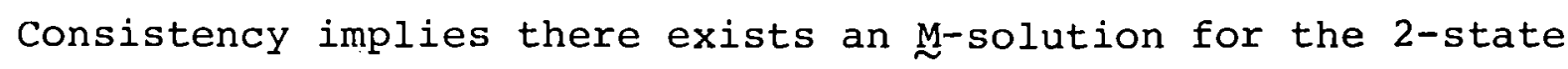
problem $(p, q)$ allocating $(a, b)$ seats respectively at $h=a+b$. This solution up to $h=a+b$ may be represented

(4)



From this we shall derive a contradiction.

Notice first, that since $\underset{M}{\mathbb{M}}$ is consistent, house-monotone and satisfies lower quota, $\underset{\sim}{M}$ is balanced. For otherwise there exists (by house monotonicity) an example in which two states have the same population $\mathrm{p}$ and apportionments $\mathrm{a}-1$ and $\mathrm{a}+1$. Hence by consistency there is an $\underset{\sim}{\mathrm{M}}$-solution for the 2-state problem $(\mathrm{p}, \mathrm{p})$ which allocates $a-1$ and $a+1$ seats respectively at $h=2 a$ but then the a - 1 allocation does not satisfy lower quota. In particular this shows $(p, a-1) \gtrless_{M}(p, a)$. 
Now consider a problem with population vector $\mathrm{p}^{\prime}=(\mathrm{p}$, $\ldots, p, q)$ having $t+1$ states, where $t$, an integer, satisfies $t \geqq q /\{q(a+1)-p(b+1)\}$. Then, we affirm, $M$ has an apportionment $(a, a, \ldots, a, b)$ for $h=t a+b$. Indeed we claim that for each $h^{\prime} \leqq t a+b$ the apportionment $\left(a^{\prime}-1, \ldots, a^{\prime}-1, a^{\prime}, \ldots, a^{\prime}, b^{\prime}\right)$, where $h^{\prime}=t a^{\prime}-t^{\prime}+b^{\prime}$, constitutes an $\underset{\sim}{M}$-solution up to $h$ having the property that the allocations to each of the pairs of states $(i, t+1),(1 \leq i \leq t)$ appears in (4)--that is, "agrees" with the solution of (4). If $h^{\prime}=0$ this is obvious. If $h^{\prime}>0$ and $t^{\prime}=0$ then by induction $\left(a^{\prime}, b^{\prime}\right)$ appears in $(4)$, so either $\left(a^{\prime}, b^{\prime}\right)=(a, b)$ and we are done, or one of $\left(a^{\prime}+1, b^{\prime}\right),\left(a^{\prime}, b^{\prime}+1\right)$ is in (4) and we can go on. If $t^{\prime}>0$ then by induction (4) has an allocation $\left(a^{\prime}-1, b^{\prime}\right)$ as well as $\left(a^{\prime}, b^{\prime}\right)$, so $\left(p, a^{\prime}-1\right) \gtrsim\left(q, b^{\prime}\right)$. But also $\left(p, a^{\prime}-1\right) \gtrsim\left(p, a^{\prime}\right)$, and therefore we can obtain an $\underset{\sim}{M}$-apportionment for $h^{\prime}+1$ seats agreeing with (4). Thus, an $\mathbb{M}$-apportionment $(a, \ldots, a, b)$ is found for $h=t a+b$. Since by hypothesis $(p, a) \gtrsim_{M}$ $(q, b),(a+1), \ldots, a+1, b)$ is an $\underset{\sim}{M}$-apportionment for $h^{*}=t(a+1)+b$. But by the choice of $t$, the exact quota of state $t+1$ at $h=h *$ satisfies

$$
\frac{q h^{*}}{t p+q}=\frac{q(t(a+1)+b)}{t p+q} \geqq \frac{q b+q+t p(b+1)}{t p+q}=b+1,
$$

contradicting the fact that $\underset{\sim}{\sim}$ satisfies lower quota. This completes the proof.

The method of smallest divisors (SD) is defined to be the set of all house-monotone solutions that yield smallest divisors apportionments. That this set is non-empty follows from remarks similar to those attendent to the monotonicity of Jefferson's 
method. SD has a symmetric relationship to $\underset{\sim}{\sim}$ and similar techniques result in mirrored results.

Theorem 2. The method of smallest divisors is the unique method that is house-monotone, consistent and satisfies upper quota.

Again, we need a lemma which is easily established by arguments similar to those used for Lemma 1.

Lemma 2. (a) Given $\underset{\sim}{\mathrm{p}}$ and $\mathrm{h}, \underset{\sim}{\mathrm{a}}=\left(\mathrm{a}_{1}, \ldots, \mathrm{a}_{\mathrm{s}}\right)$ is a smallest divisors apportionment for $h$ if and only if

$$
\max _{i} p_{i} / a_{i} \leqq \min _{i} p_{i} /\left(a_{i}-1\right)
$$

(b) The set of smallest divisors solutions is the set of all solutions $\underset{\sim}{f}$ obtained recursively as follows:

(i) $\quad \underset{\sim}{(p}, 0)=0$

(ii) if $a_{i}=f_{i}(p, h)$ is an apportionment for $h$ and $k$ is some one state for which $p_{k} / a_{k}=\max _{i} p_{i} / a_{i}$, then

$$
\mathrm{f}_{\mathrm{k}}(\underset{\sim}{\mathrm{p}}, \mathrm{h}+1)=\mathrm{a}_{\mathrm{k}}+1, \quad \mathrm{f}_{i}(\underset{\sim}{\mathrm{p}}, \mathrm{h}+1)=\mathrm{a}_{i} \text { for } i \neq k \text {. }
$$

The proof of Theorem 2 parallels that of Theorem 1.

SD is clearly consistent and house-monotone by Lemma 2 . To see it satisfies upper quota, suppose not. Then for some apportionment $\underset{\sim}{\text { for }} \mathrm{h}$ we have $a_{i}>\left[q_{i}\right]$, that is $a_{i} \geqq q_{i}+1$. 
Then for some state $j \neq i$ we must have $a_{j}<q_{j}$. Letting $\lambda=\Sigma_{k} p_{k} / h$ we have then that $q_{i}=p_{i} / \lambda, q_{j}=p_{j} / \lambda$ and

$$
p_{j} / a_{j}>\lambda \geqq p_{i} /\left(a_{i}-1\right)
$$

contradicting Lemma $2(a)$.

Conversely, suppose that $\underset{\sim}{\mathbb{M}}$, consistent, house-monotone and satisfying upper quota, is not SD. Then there exist populations $(p, q)$ having $\underset{\sim}{M}$ allocations $a, b$ which are $\underset{\sim}{S}$ allocations but

$$
(p, a) \geq(q, b) \text { whereas } p / a<q / b \text {. }
$$

From this we derive a contradiction.

By arguments identical to those used in proving Theorem 1 , $\underset{\sim}{M}$ has an apportionment $(a, b, b, \ldots, b)$ for $h=a+t b$, for $a$ problem with $t+1$ states and populations $(p, q, \ldots, q)$, where $t$, integer, satisfies $t \geqq p /\{a q-b p\}$. By consistency $(a+1, b, \ldots, b)$ is an $\underset{\sim}{M}$-apportionment for $h=h *=a+t b+1$. But, by the choice of $t$, the exact quota of the first state at $h=h *$ satisfies

$$
\frac{p h *}{p+t q}=\frac{p(a+t b+1)}{p+t b} \leqq \frac{p a+t q a}{p+t q}=a
$$

contradicting the fact that $\underset{\sim}{\mathbb{M}}$ satisfies upper quota. This completes the proof. 


\section{Satisfying Quota}

A method is said to satisfy quota if it satisfies both lower quota and upper quota. It turns out that no monotone and consistent method satisfies quota [2], whence, in particular, $\mathrm{Z}$ does not satisfy upper quota and SD does not satisfy lower quota. of course, the Hamilton method does satisfy quota; however it is neither consistent nor monotone.

Proportionality, or "according to respective numbers," means ideally, attributing to each state its exact quota. But rational allocations are not permitted, integers are required. Historical precedent, beginning with the deliberations at the Constitutional Convention, establishes the need for satisfying quota. Further, any apportionment not satisfying quota has been [9], and would be, found by the ordinary citizen to be contrary to common sense. Second, ever since the recognition of the Alabama paradox, house monotonicity has, by Congress, been taken as an essential property. Therefore, it is natural to ask if there does exist a method which satisfies quota and is house-monotone. The answer is yes. If consistency is weakened to apply only when quota is not violated, then there is a method, called the quota method, which is the unique method satisfying these properties [1,3]. Thus, Hamilton's principle of satisfying quota is reconciled with Jefferson's approach. 


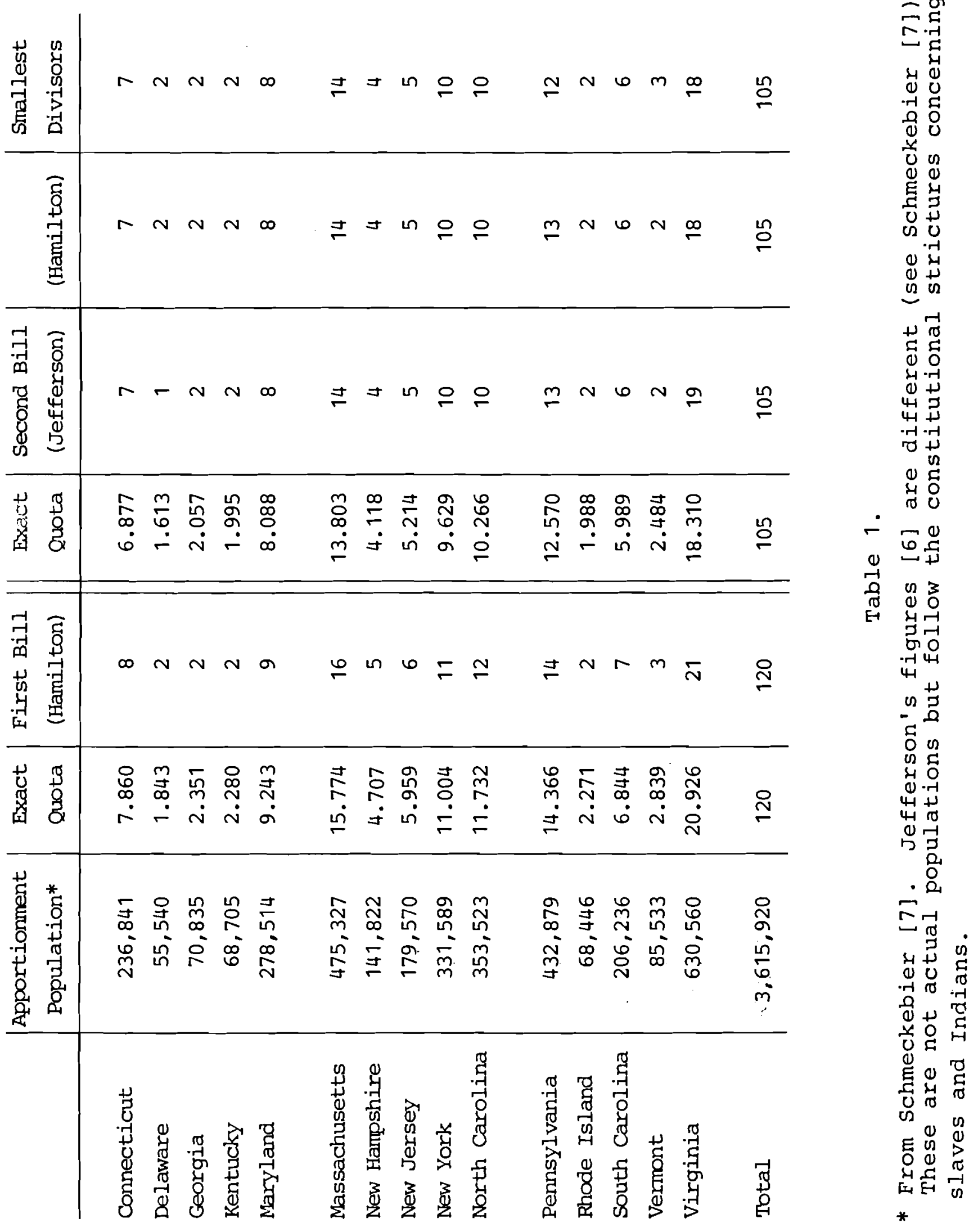


[1] Balinski; M.L., and H.P. Young, A New Method for Congressional Apportionment, Proceedings of the National Academy of Sciences, U.S.A., 71, (1974), 4602-4606.

[2] Criteria for Proportional Representation, submitted for publication.

[3] - The Quota Method of Apportionment, American Mathematical Monthly, 82 , (1975), 701-730.

[4] , On Huntington Methods of Apportionment, submitted for publication.

[5] Hamilton, Alexander, The Papers of Alexander Hamizton, Vol. XI (Eebruary 1792-June 1792), Harold C. Syrett, ed., Columbia University Press, New York 1966, 228-230.

[6] Jefferson, Thomas, The Works of Thomas Jefferson, Vol. VI, Paul Leicester Ford, ed., G.P.'Putnam and Sons, New York 1904, 460-471.

[7] Schmeckebier, Laurence F., Congressional Apportionment, The Brookings Institution, Washington, D.C. 1941.

[8] Washington, George, The Writings of George Washington, Vol. 32 (March 10, 1792-June 30, 1793), John C. Fitzpatrick, ed., United States Government Printing office, Washington, D.C. 1941.

[9] Webster, Daniel, The Writings and Speeches of Daniel Webster, Vol. VI, National Edition, Little, Brown and Company, Boston, Massachusetts 1903, 102-123. 